\title{
Exacerbation of signs and symptoms of allergic conjunctivitis by a controlled adverse environment challenge in subjects with a history of dry eye and ocular allergy
}

\author{
This article was published in the following Dove Press journal: \\ Clinical Ophthalmology \\ 18 January 2013 \\ Number of times this article has been viewed
}

\author{
Paulo J Gomes' \\ George W Ousler' \\ Donna L Welch' \\ Lisa M Smith' \\ Jeffrey Coderre' \\ Mark B Abelson ${ }^{1,2}$ \\ 'Ora Inc, Andover, MA; ${ }^{2}$ Harvard \\ Medical School, Schepens Eye \\ Research Institute, Boston, MA, USA
}

Correspondence: Paulo J Gomes

Ora Inc, 300 Brickstone Square,

Andover, MA 01810, USA

Tel +l 9786858900

Email pgomes@oraclinical.com
Background: The goal of this study was to assess the effect of a controlled adverse environment (CAE) challenge on subjects with both allergic conjunctivitis and dry eye.

Methods: Thirty-three subjects were screened and 17 completed this institutional review board-approved study. Subjects underwent baseline ocular assessments and conjunctival allergen challenge $(\mathrm{CAC})$ on days 0 and 3 . Those who met the ocular redness and itching criteria were randomized to receive either the controlled adverse environment (CAE) challenge (group A, $\mathrm{n}=9$ ) or no challenge (group $\mathrm{B}, \mathrm{n}=8$ ) at day 6 . Thirty minutes after CAE/no-CAE, subjects were challenged with allergen and their signs and symptoms graded. Exploratory confocal microscopy was carried out in a subset of subjects at hourly intervals for 5 hours post-CAC on days 3 and 6.

Results: Seven minutes post-CAC, subjects exposed to the CAE had significantly greater itching (difference between groups, $0.55 \pm 0.25, P=0.028)$, conjunctival redness $(0.59 \pm 0.19$, $P=0.002)$, episcleral redness $(0.56 \pm 0.19, P=0.003)$ and mean overall redness (mean of conjunctival, episcleral, and ciliary redness, $0.59 \pm 0.14, P<0.001)$. The mean score at 7,15 , and 20 minutes post-CAC for conjunctival redness $(0.43 \pm 0.17, P=0.012)$, episcleral redness $(0.49 \pm 0.15, P=0.001)$, mean overall redness in all regions $(0.43 \pm 0.15, P=0.005)$, and mean chemosis $(0.20 \pm 0.08, P=0.017)$ were also all significantly greater in CAE-treated subjects. Confocal microscopic images of conjunctival vessels after CAC showed more inflammation in CAE-treated subjects.

Conclusion: In subjects with both dry eye and allergic conjunctivitis, exposure to adverse environmental conditions causes an ocular surface perturbation that can intensify allergic reactions

Keywords: allergic conjunctivitis, dry eye, conjunctival allergen challenge, controlled adverse environment, comorbidity

\section{Introduction}

Allergy is described as the fifth leading group of chronic diseases, affecting 50 million Americans. The Third National Health and Nutrition Examination Survey recently reported that $40 \%$ of the population had episodes of ocular allergy. ${ }^{1}$ Dry eye disease has a prevalence estimated at 5\%-30\% of the general population, with variations according to age and gender. ${ }^{2}$ In a survey by the American Academy of Ophthalmology, respondents reported that approximately 30\% of patients seeking treatment at an ophthalmologist's office have symptoms consistent with dry eye disease. ${ }^{3}$ It has been 
estimated that in the US, approximately 5 million people 50 years and older have moderate to severe dry eye. ${ }^{4}$

The magnitudes of these reported incidences of allergic conjunctivitis and dry eye disease suggest that there could be significant comorbidity. 5,6 The variable nature of dry eye pathophysiology has in large part prevented epidemiological studies on its coexistence in patients with allergic conjunctivitis. Dry eye and allergic conjunctivitis share signs and symptoms, complicating a differential diagnosis. ${ }^{5,6}$ Inflammation is a key feature in dry eye, ${ }^{7}$ and is known to be an important component of chronic ocular allergy. ${ }^{8-11}$ The tear film insufficiency and ocular surface inflammation present in dry eye disease might be expected to facilitate allergen entry and exacerbate allergic ocular signs and symptoms in a sensitized individual, but no studies have yet shown this association.

The objective of the current study was to determine whether subjects with a history of both dry eye and ocular allergy would show a heightened reaction to an allergen challenge following an adverse environmental exposure.

\section{Materials and methods Study design}

This study was conducted in accordance with institutional review board (Alpha IRB, San Clemente CA) regulations and with the ethical principles that originated with the Declaration of Helsinki. Written informed consent was obtained from each subject prior to any study procedures.

\section{Subject inclusion and exclusion criteria}

Subjects were $\geq 18$ years of age and had to have a diagnostic skin test indicative of allergy to cat hair or dander, grasses, ragweed, dust mites, dog dander, cockroach, or tree antigens, as well as a history of seasonal or perennial allergic conjunctivitis for at least one year prior to visit 1 . Subjects also had to have a diagnosis of dry eye and a history of use or a desire to use tear substitutes in the previous 6 months, as well as a total corneal fluorescein staining score of $\geq 2$ on a predefined Ora Calibra ${ }^{\mathrm{TM}}$ scale from 0 to 4 , with $0=$ none and $4=$ worst in at least one eye at visit 1 . All subjects had to have a best corrected visual acuity of +0.7 or better assessed by the Early Treatment of Diabetic Retinopathy Study scale in both eyes at visit 1 .

Subjects were excluded if they had: any significant ocular infection or inflammation other than dry eye; ocular surgery within 3 months prior to visit 1 or refractive surgery within 6 months; any history of retinal detachment, diabetic retinopathy, or progressive retinal disease; or any history of anaphylactic reaction to any allergens used in this study or any systemic disease or uncontrolled medical condition that in the opinion of the investigator could have interfered with the study. Contact lens wear was prohibited up to 7 days prior to visit 1 , as well as use of the following medications (washout period prior to visit 1 in parentheses): systemic or ocular $\mathrm{H} 1$ antihistamines or $\mathrm{H} 1$ antihistamine-vasoconstrictor drug combinations (72 hours); decongestants, monoamine oxidase inhibitors, artificial tears, or lid scrubs, mast cell stabilizers, prostaglandins or prostaglandin derivatives, and ocular, topical or systemic nonsteroidal anti-inflammatory drugs (7 days); inhaled, ocular, or topical corticosteroids (14 days), depot corticosteroids (45 days), all other topical ophthalmic preparations (72 hours), and lastly, no change in immunotherapy for at least 2 months prior to and during the study. Subjects could have withdrawn from the study at any time, and the investigator could have discontinued any subject at any time for safety reasons.

The conjunctival allergen challenge (CAC) model ${ }^{12,13}$ was used to select subjects who had a reproducible and predefined response to the allergen challenge. Those subjects who responded positively to CAC must also have reported a history of dry eye. Eligible subjects were then randomized into two groups in a masked fashion. Prior to CAC, half of the subjects (group A, CAE + CAC), were exposed for 90 minutes to a controlled adverse environment $\left(\mathrm{CAE}^{\mathrm{SM}}\right)$ chamber, ${ }^{14-16}$ a dry eye research model that exacerbates signs and symptoms of dry eye in a controlled manner by regulating humidity, temperature, airflow, lighting conditions, and visual tasking. The second half of the subjects (group B, CAC only) waited for 90 minutes in a normal environment and had signs and symptoms of dry eye assessed in a manner identical to group A. Both groups then received the CAC 30 minutes after completion of the CAE exposure. The magnitude of the allergic responses was compared.

The study comprised three visits over a 6-day period:

- visit 1 (day 0) screening, allergen titration

- visit 2 (day 3) allergen confirmation CAC, enrollment, randomization

- visit 3 (day 6) CAE exposure followed by repeat CAC (Figure 1).

\section{Efficacy and safety measures}

Subject assessment of ocular itching employed the Ora Calibra itch scale, a $0-4$ scale where $0=$ none and $4=$ incapacitating. Subjects also graded tearing, eyelid swelling, ocular discomfort, a four-symptom questionnaire, and nasal signs and symptoms using 0-4 scales. The investigator graded 


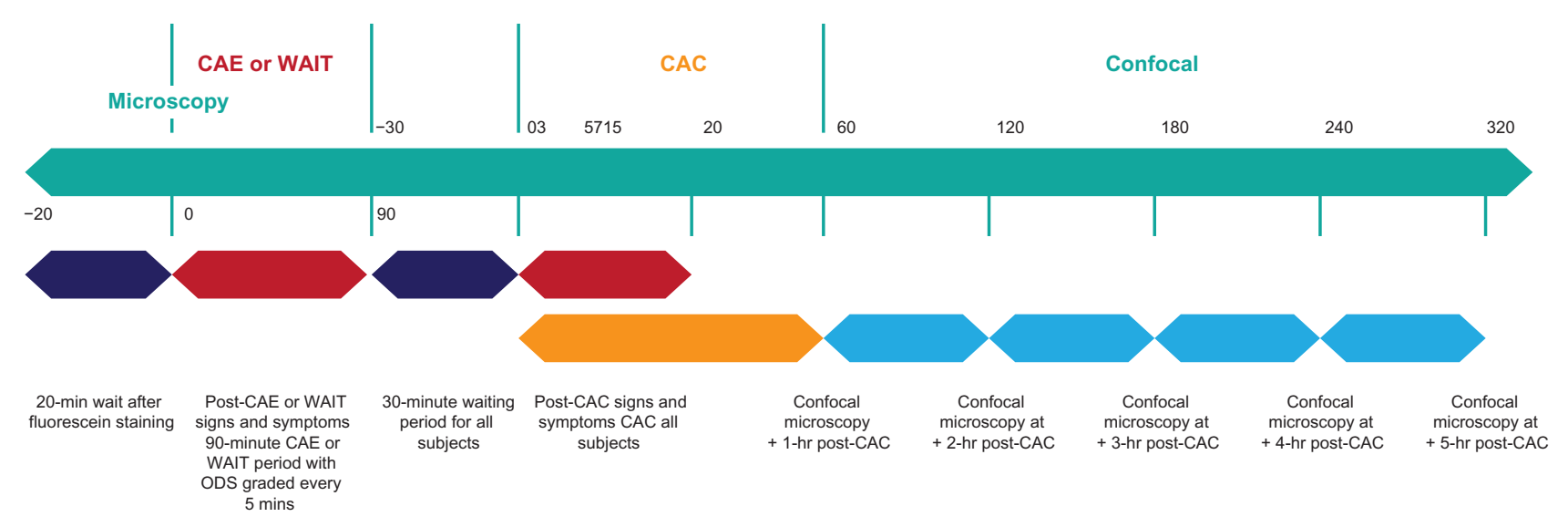

Figure I Study visit 3 diagram.

ocular redness using the Ora Calibra redness scale (conjunctival, episcleral, ciliary; $0=$ none to $4=$ extremely severe) and chemosis at 7, 15, and 20 minutes post-CAC (0-4 scale). Safety parameters were pregnancy testing, slit lamp biomicroscopy, visual acuity, and adverse event reporting.

\section{Exploratory measures}

Confocal microscopy (Heidelberg Retinal Tomography II, Heidelberg Engineering, Carlsbad, CA) was used to visualize superficial conjunctival blood vessels as an indicator of the allergic reaction. Images were obtained at visit 1 (baseline) and post-CAC at visits 2 and 3. The images were evaluated using the Ora Calibra conjunctival inflammation confocal scale (patent pending), a 0-4 scale to grade for leukocyte presence and adhesion in and around the conjunctival blood vessels.

\section{Screening and allergen titration}

At visit 1, demographic data and medical histories were recorded and medication washout periods were determined to be adequate. A urine pregnancy test was given to women of childbearing potential. The following procedures were carried out: best corrected visual acuity (eligible subjects had a $\log$ MAR score of 0.70 or better in each eye), subjective ocular discomfort score and four-symptom questionnaire, ocular and nasal allergic signs and symptoms, slit-lamp biomicroscopy, tear film break-up time (TFBUT), fluorescein corneal staining, and baseline confocal biomicroscopy.

Twenty minutes after baseline confocal microscopy, the titration CAC was carried out bilaterally with one allergen to which the subject was sensitized (cat dander, dog dander, cockroach, dust mite, meadow fescue, Kentucky bluegrass, ragweed, or timothy grass). One drop of solubilized allergen at the weakest dilution was instilled bilaterally into the
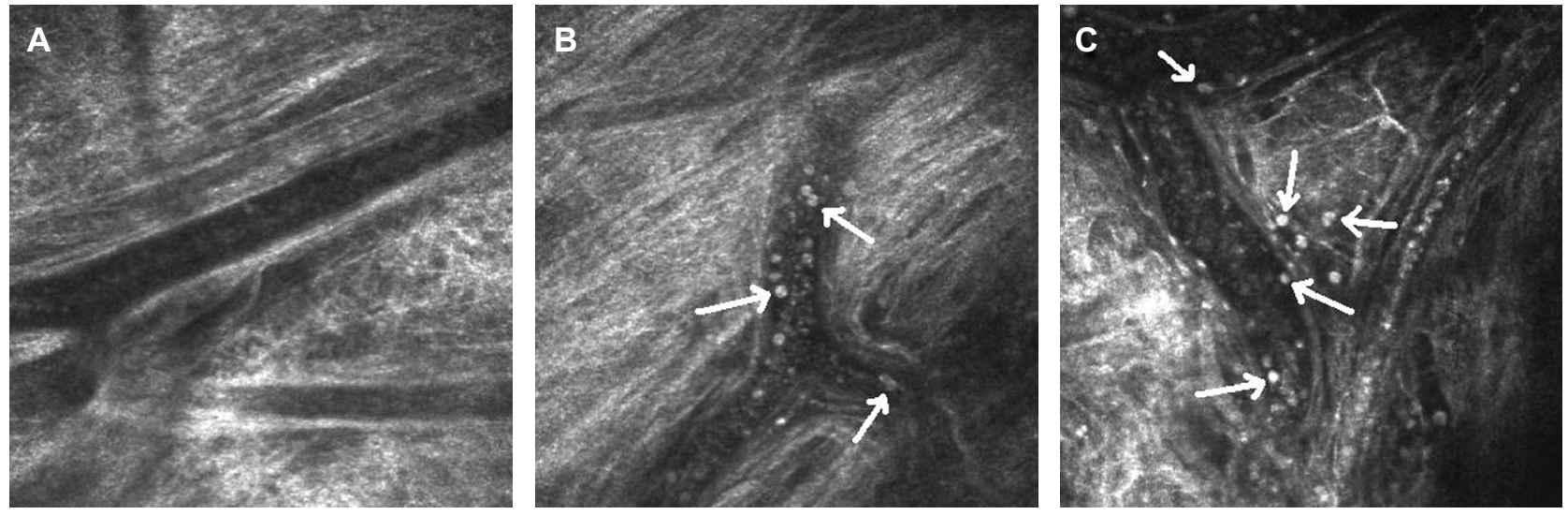

Figure 2 Representative frames from confocal video-microscopy of the bulbar conjunctiva. (A) Image captured prior to treatments. Note that longitudinal vessels contain translucent redness cells, and no white cells are visible. (B) Image captured one hour following allergen challenge; white cells are visible within the vasculature; several appear to be either adhering to vessel wall or in process of diapedesis. (C) Image captured one hour following allergen challenge in subject exposed to CAE ( 90 minutes) prior to challenge.

Note: White cells are visible in the vasculature and white cell infiltration into tissues surrounding vessels is extensive. 
conjunctival cul de sac. If the subject failed to react within ten minutes, increasingly concentrated doses were instilled bilaterally at 10-minute intervals until a positive reaction was elicited. If a positive reaction was not elicited with the first allergen, other allergens to which the subject's skin test was positive could have been used. A positive CAC reaction was defined as a score of $\geq 2$ for ocular itching and $\geq 2$ for conjunctival redness in both eyes within 10 minutes of receiving that dose of allergen. Any subject who failed to test positively was excluded from the study. Post-CAC ocular and nasal signs and symptoms were assessed. Any subject who experienced discomfort from the allergic reaction was given a commercially available antiallergy relief medication (Visine- $\mathrm{A}^{\circledR}$ ) after the ocular allergy endpoints were assessed and before exiting the clinic.

\section{Allergen confirmation}

At visit 2, medical and medication histories were updated and subjects were asked if any adverse event had occurred after visit 1. Best corrected visual acuity, ocular discomfort, four-symptom questionnaire (Ora Calibra four-symptom assessment questionnaire grading burning, dryness, grittiness, stinging on a $0-5$ scale where $0=$ none and $5=$ worst), ocular and nasal allergic signs and symptoms, slit lamp biomicroscopy, TFBUT, and corneal fluorescein staining were carried out.

At least 15 minutes after fluorescein staining, the CAC was then repeated bilaterally using the same dose of allergen that elicited a positive response at visit 1 . Subjects assessed ocular itching at 3,5, and 7 minutes post-CAC and graded tearing, eyelid swelling, and nasal signs and symptoms at 7, 15, and 20 minutes post-CAC. The investigator graded ocular redness (conjunctival, episcleral, ciliary) and chemosis at 7, 15, and 20 minutes post-CAC. Subjects had to react with $a \geq 2$ in itching and redness at two of these three corresponding post-CAC time points to be eligible to continue in the study.

Eligible subjects were randomized into two groups: group A, to undergo the 90-minute CAE challenge at visit 3 (day 6) prior to a repeat $\mathrm{CAC}$ and group $\mathrm{B}$, to wait in a normal environment for the same 90-minute period prior to a repeat CAC. Subjects were further randomized into five subgroups for confocal microscopy at 1, 2, 3, 4, and 5 hours post-CAC at visits 2 and 3 . After confocal microscopy, relief medication was provided if necessary.

\section{$\mathrm{CAE}$ followed by conjunctival allergen challenge}

At visit 3, medical and medication histories were updated and subjects were asked if any adverse event had occurred after visit 2. BCVA, ocular discomfort, four-symptom questionnaire, ocular and nasal allergic signs and symptoms, slit-lamp biomicroscopy, TFBUT, and corneal fluorescein staining were carried out.

Twenty $( \pm 5)$ minutes after fluorescein staining, group A subjects underwent the 90-minute CAE exposure. Both groups graded ocular discomfort at time 0 and every 5 minutes ( \pm one minute) thereafter for 90 minutes. Post-CAE, slit lamp biomicroscopy, TFBUT, and fluorescein staining were performed.

Within 30 minutes of exiting the CAE, a CAC was administered to both groups at the same allergen dose that elicited a positive reaction at visit 2. Post-CAC ocular and nasal signs and symptoms were assessed. Subjects then waited their specified time $(1,2,3,4$, or 5 hours postCAC), before undergoing confocal microscopy. Adverse events were assessed and relief medication provided at the subject's request. Subjects were then exited from the study.

\section{Statistical methods}

The primary efficacy variables were ocular itching evaluated by the subject at 3,5 , and 7 minutes post-CAC and conjunctival redness evaluated by the investigator at 7,15 , and 20 minutes post-CAC at visit 3 . The secondary efficacy variables were ciliary and episcleral redness and chemosis graded by the investigator at 7, 15, and 20 minutes post-CAC and eyelid swelling, tearing, and nasal signs and symptoms evaluated by the subject at 7 , 15 , and 20 minutes, as well as inferior corneal fluorescein staining at visit 3. Mean scores at all time points post-CAC were also calculated, as well as mean scores in all three redness regions.

Continuous variables were summarized using descriptive statistics, including means, medians, standard deviations, minimums, and maximums. Categorical variables were summarized with frequencies and percentages. Statistical analyses were performed with SAS version 9.2 (SAS Institute Inc, Cary, NC).

It was hypothesized that eyes would have shown an increase in post-CAC ocular itching and/or conjunctival redness at any post-CAC assessment after having been exposed to the CAE for 90 minutes. This hypothesis was assessed by comparing mean scores for groups A and B. The 17 with allergic conjunctivitis subjects and dry eye gave a power of $75 \%$ to detect, at the $5 \%$ level, a group difference of 0.75 units in mean ocular itching or mean conjunctival redness. This calculation assumed that the 
common standard deviation of scores averaged over the eyes was 0.75 .

The primary and secondary efficacy parameters were analyzed by a repeated-measurement normal linear model estimated by generalized estimating equation methods. Wald tests based on this model were two-sided and used significance levels of 5\%. Estimates, tests, and confidence intervals were made with and without adjustment for visit 2 baseline scores.

Confocal microscopy profiles were estimated as a function of time from exiting the CAC. For broad statistical comparisons, the mean of the inflammation scores over the 5-hour period was calculated and the two groups, with and without CAE pretreatment, were compared.

\section{Results}

\section{Demographic data}

Thirty-three subjects were screened on visit 1. Of these, 11 failed to meet all inclusion and exclusion criteria. Twenty-two subjects returned for visit 2 and five of these failed to have a sufficient $\mathrm{CAC}$ response and were thus eliminated from the study. The final numbers of subjects who completed the study were nine in group $\mathrm{A}(\mathrm{CAE}+\mathrm{CAC})$ and eight in group $\mathrm{B}$ (CAC only).

Group A had a mean age of 55.22 \pm 12.01 years, with a range from 26 to 68 and a median of 58 years. Group B had a mean age of $63.50 \pm 7.73$ years, with a range from 56 to 75 and a median of 61 years. These differences were not statistically significant $(P=0.096)$. Group A was comprised of four males and five females and group B of five males and three females. All subjects were Caucasian. No protocol deviations or adverse events occurred during the study.

\section{Effects of CAE on dry eye signs}

\section{and symptoms}

Table 1 provides data that confirmed the effects of the CAE challenge on dry eye/allergic subjects (group A) compared with similar subjects who waited the 90-minute period in a normal environment (group B). After a CAE challenge, mean ( \pm standard error) superior fluorescein staining scores were $1.92 \pm 0.30$ for group A and $1.38 \pm 0.28$ for group B. After adjustment for baseline, this $0.68 \pm 0.29$ difference was statistically significant $(P=0.019)$. The total mean fluorescein staining score was almost significantly different between groups, at $1.47 \pm 0.25$ for group A versus $1.13 \pm 0.23$ for group B, with an adjusted for baseline mean difference of $0.53 \pm 0.27(P=0.052)$.

These staining differences were also reflected in differences in ocular discomfort scores. After a 90-minute CAE, mean ocular discomfort scores were $3.50 \pm 0.17$ for group $\mathrm{A}$ and $1.31 \pm 0.55$ for group $\mathrm{B}$, representing a mean difference of $2.19 \pm 0.55(P<0.001)$. After adjustment for baseline, this mean difference of $2.11 \pm 0.60$ was still significant $(P<0.001)$. This was also the case for mean ocular discomfort scores graded during the CAE every 5 minutes from 5 to 90 minutes (a mean of 18 assessments). Without adjustment for baseline, mean overall discomfort was $3.01 \pm 0.20$ for group A and $1.24 \pm 0.36$ for group B, a highly significant difference of $+1.77 \pm 0.40(P<0.001)$. After adjustment for baseline, the mean difference was still significant at $1.58 \pm 0.41(P<0.001)$. These results confirmed the effects of a 90-minute CAE challenge on the signs and symptoms of dry eye in a population of dry eye/ allergic subjects. There was no significant difference in TFBUT between these two groups, ie, the CAE did not have

Table I Effects of a CAE challenge in predefined subjects with a history of dry eye and allergic conjunctivitis (group A, CAE + CAC) compared with similar subjects who remained for the 90-minute challenge period in a normal environment (group B, CAC only)

\begin{tabular}{|c|c|c|c|c|c|c|}
\hline Sign or symptom of dry eye & $\begin{array}{l}\text { Group A } \\
\text { CAE } \\
\text { Mean } \pm \text { SE } \\
(n=9)\end{array}$ & $\begin{array}{l}\text { Group B } \\
\text { No CAE } \\
\text { Mean } \pm \text { SE } \\
(n=8)\end{array}$ & $\begin{array}{l}\text { Mean difference } \\
\text { unadjusted for } \\
\text { baseline } \pm \text { SE }\end{array}$ & $P$ value & $\begin{array}{l}\text { Mean difference } \\
\text { adjusted for } \\
\text { baseline } \pm \text { SE }\end{array}$ & $P$ value \\
\hline Central fluorescein staining & $0.61 \pm 0.23$ & $0.41 \pm 0.20$ & $0.20 \pm 0.31$ & 0.505 & $0.32 \pm 0.33$ & 0.336 \\
\hline Inferior fluorescein staining & $1.89 \pm 0.29$ & $1.59 \pm 0.28$ & $0.30 \pm 0.40$ & 0.465 & $0.34 \pm 0.29$ & 0.242 \\
\hline Superior fluorescein staining & $1.92 \pm 0.30$ & $1.38 \pm 0.28$ & $0.54 \pm 0.41$ & 0.190 & $0.68 \pm 0.29$ & 0.019 \\
\hline Mean fluorescein staining & $1.47 \pm 0.25$ & $1.13 \pm 0.23$ & $0.35 \pm 0.34$ & 0.313 & $0.53 \pm 0.27$ & 0.052 \\
\hline $\begin{array}{l}\text { Ocular discomfort scores at } 90 \text { minutes } \\
\text { post-CAE }\end{array}$ & $3.50 \pm 0.17$ & $I .31 \pm 0.55$ & $2.19 \pm 0.55$ & $<0.001$ & $2.11 \pm 0.60$ & $<0.001$ \\
\hline $\begin{array}{l}\text { Ocular discomfort scores during CAE } \\
\text { (mean of } 18 \text { scores from 5-90 minutes) }\end{array}$ & $3.01 \pm 0.20$ & $1.24 \pm 0.36$ & $\mathrm{I} .77 \pm 0.40$ & $<0.001$ & $|.58 \pm 0.4|$ & $<0.001$ \\
\hline TFBUT & $2.83 \pm 0.39$ & $2.89 \pm 0.30$ & $-0.06 \pm 0.50$ & 0.904 & $0.09 \pm 0.47$ & 0.855 \\
\hline
\end{tabular}

Note: These results confirmed the exacerbating effect of CAE on dry eye signs and symptoms.

Abbreviations: CAE, controlled adverse environment; CAC, conjunctival allergen challenge; SE, standard error of the mean; TFBUT, tear film break-up time. 
Table 2

\begin{tabular}{|c|c|c|c|c|c|c|c|}
\hline \multirow{2}{*}{$\begin{array}{l}\text { Sign/symptom/ } \\
\text { time post-CAC }\end{array}$} & \multicolumn{2}{|c|}{ Visit 2 (day 3) (baseline CAC) } & \multicolumn{5}{|c|}{ Visit 3 (day 6$)$ ( \pm CAE exposure 30 minutes prior to CAC) } \\
\hline & $\begin{array}{l}\text { Group A } \\
\text { sample mean } \\
\pm S E(n=9)\end{array}$ & $\begin{array}{l}\text { Group B } \\
\text { sample mean } \\
\pm \text { SE }(n=8)\end{array}$ & $\begin{array}{l}\text { Group A } \\
\text { (CAE) adjusted from } \\
\text { baseline model means }\end{array}$ & $\begin{array}{l}\text { Group B } \\
\text { (no CAE) adjusted } \\
\text { from baseline model } \\
\text { means }\end{array}$ & $\begin{array}{l}\text { Mean } \\
\text { difference } \\
\pm \mathrm{SE}^{\mathrm{b}}\end{array}$ & $95 \% \mathrm{Cl}$ & $P$ value ${ }^{c}$ \\
\hline \multicolumn{8}{|l|}{ Ocular itching } \\
\hline Pre-CAC & $0.50 \pm 0.21$ & $0.72 \pm 0.35$ & 0.39 & 0.06 & $0.33 \pm 0.15$ & $0.04-0.62$ & 0.024 \\
\hline 3 minutes & $2.42 \pm 0.32$ & $2.31 \pm 0.22$ & 1.97 & 2.29 & $\begin{array}{l}-0.32 \pm \\
0.42\end{array}$ & $-1.15-0.50$ & 0.444 \\
\hline 5 minutes & $2.89 \pm 0.29$ & $2.47 \pm 0.21$ & 2.64 & 2.62 & $0.02 \pm 0.39$ & $-0.74-0.77$ & 0.968 \\
\hline 7 minutes & $2.89 \pm 0.29$ & $2.56 \pm 0.22$ & 3.15 & 2.61 & $0.55 \pm 0.25$ & $0.06-1.03$ & 0.028 \\
\hline $\begin{array}{l}\text { Average of } 3,5,7 \\
\text { minutes }\end{array}$ & $2.73 \pm 0.28$ & $2.45 \pm 0.21$ & 2.59 & 2.50 & $0.08 \pm 0.33$ & $-0.57-0.73$ & 0.801 \\
\hline \multicolumn{8}{|l|}{ Conjunctival } \\
\hline Pre-CAC & $0.86 \pm 0.26$ & $0.50 \pm 0.09$ & 0.81 & 0.62 & $0.19 \pm 0.35$ & $-0.5 \mid-0.88$ & 0.596 \\
\hline 7 minutes & $1.78 \pm 0.13$ & $2.19 \pm 0.14$ & 1.89 & 1.31 & $0.59 \pm 0.19$ & $0.21-0.96$ & 0.002 \\
\hline 15 minutes & $2.06 \pm 0.15$ & $2.53 \pm 0.12$ & 2.00 & 1.75 & $0.25 \pm 0.14$ & $-0.02-0.52$ & 0.066 \\
\hline 20 minutes & $2.22 \pm 0.11$ & $2.78 \pm 0.14$ & 2.00 & 1.71 & $0.29 \pm 0.24$ & $-0.18-0.76$ & 0.222 \\
\hline $\begin{array}{l}\text { Average of } 7,15, \\
20 \text { minutes }\end{array}$ & $2.02 \pm 0.12$ & $2.50 \pm 0.12$ & 1.99 & 1.56 & $0.43 \pm 0.17$ & $0.10-0.77$ & 0.012 \\
\hline
\end{tabular}

an adverse effect on TFBUT in this study. Mean TFBUT was $2.83 \pm 0.39$ in group A and $2.89 \pm 0.30$ in group $B$, representing a nonsignificant difference of $-0.06(P=0.904)$. After adjustment for baseline, this mean difference was still not significant $(0.09 \pm 0.47, P=0.855)$.

\section{Effects of CAE on conjunctival allergen challenge response}

Primary endpoints

Significant differences between group A $(\mathrm{CAE}+\mathrm{CAC})$ and group $\mathrm{B}$ (CAC only) were noted in the primary endpoints of ocular itching and conjunctival redness post-CAC. Table 2 shows the ocular itching and conjunctival redness data for the visit 2 baseline $\mathrm{CAC}$ and visit 3 (CAC $\pm \mathrm{CAE}$ ) assessments. At baseline, pre-CAC, ocular itching was significantly greater in CAE-exposed subjects (mean difference between groups, $0.33 \pm 0.15, P=0.024)$. At 7 minutes after $\mathrm{CAC}$, both ocular itching (mean difference between groups, $0.55 \pm 0.25, P=0.028$ ) and conjunctival redness (mean difference between groups, $0.59 \pm 0.19$, $P=0.002$ ) were greater in CAE-exposed subjects. The overall mean post-CAC conjunctival redness score (mean of the 7 , 15 , and 20 minutes post-CAC scores) was also significantly higher in CAE-exposed subjects (mean difference between groups, $0.43 \pm 0.17, P=0.012$ ).

\section{Secondary endpoints}

Increased redness was observed in the secondary endpoints in the CAE-exposed subjects (see Table 3). At 7 minutes after CAC, both episcleral redness (mean difference between groups, $0.56 \pm 0.19, P=0.003)$ and mean redness in all regions (conjunctival, ciliary, episcleral; mean difference between groups, $0.59 \pm 0.14, P<0.001$ ) were greater in CAE-exposed subjects. The mean post-CAC episcleral redness score (mean of the 7,15 , and 20 minute post-CAC scores) was also significantly greater in CAE-exposed subjects (mean difference between groups, $0.49 \pm 0.15, P=0.001)$. There were no increases observed in ciliary redness. The mean post-CAC chemosis score (mean of the 7, 15, and 20 minutes post-CAC scores) was also significantly greater in CAE-exposed subjects (mean difference between groups $0.20 \pm 0.08, P=0.017$ ). None of the subject-graded nasal symptom (rhinorrhea, nasal pruritus, nasal congestion, ear and palate pruritus) scores were significantly different between the two groups.

\section{Exploratory endpoint: confocal microscopy}

The visit 1 baseline HRT II confocal microscopy scores represent conjunctival vessels in a quiescent state before any $\mathrm{CAE}$ or CAC challenges. Confocal microscopic evaluation of conjunctival vessels revealed post-CAC inflammatory changes compared with baseline. 


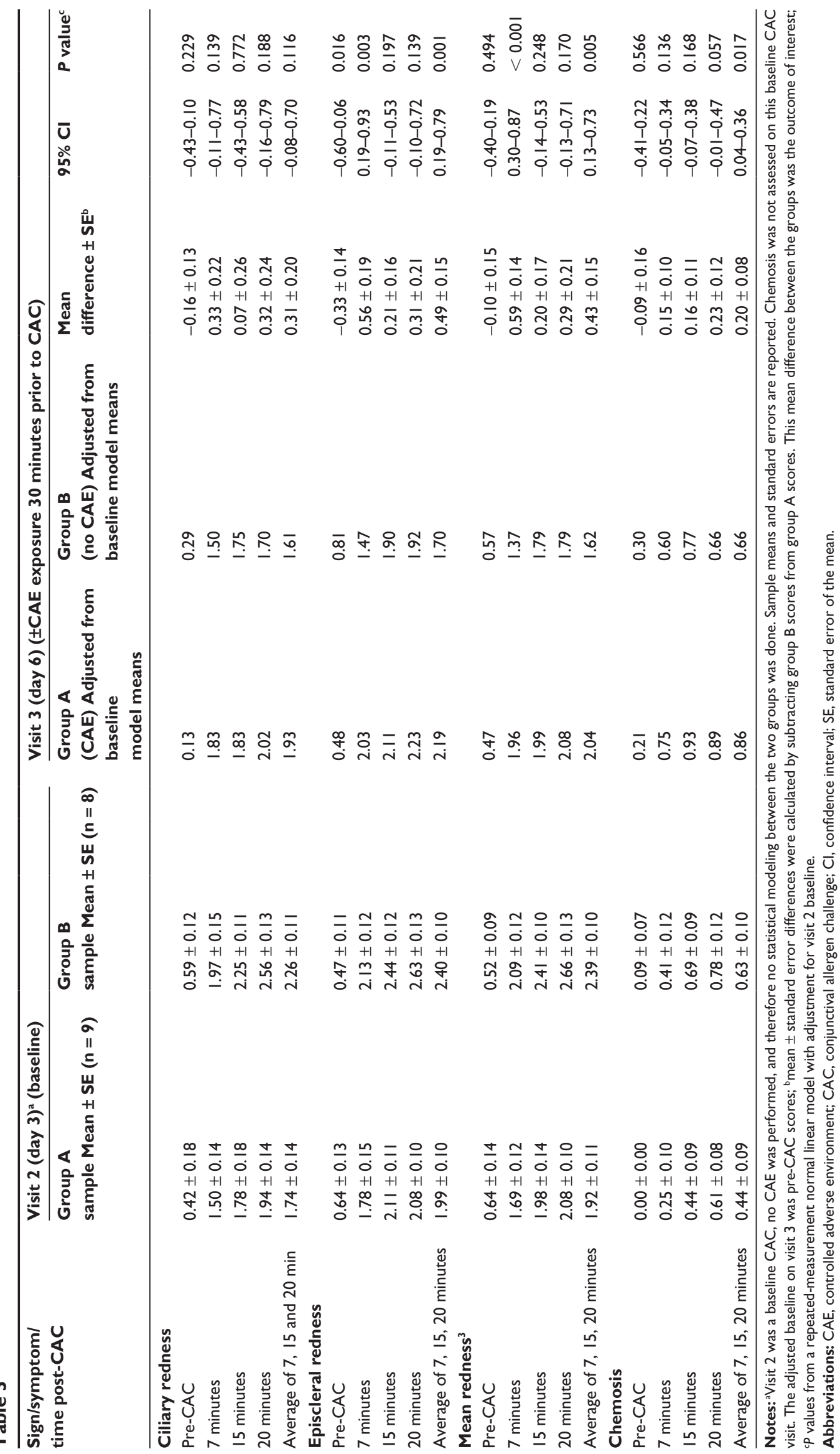


The visit 1 mean inflammation score for all subjects at baseline was $1.24 \pm 0.24(n=17)$. The visit 2 post-CAC mean inflammation score for all subjects was $2.53 \pm 0.17$ at the mean time after CAC (3.06 hours, $\mathrm{n}=17$ ). The post-CAC inflammation scores at visits 2 and 3 tended to be higher at one hour post-CAC and to decay over time out to 5 hours. The numbers of subjects at each of the five hourly post-CAC time points at visits 2 and 3 were small $(n=1$ or 2$)$, therefore a mean value was calculated for the inflammation score at the mean time post-CAC. At visit 3, differences in inflammation scores between group A and group B were significantly different. The mean inflammation scores over the five-hour period were 3.16 in group A $(n=6)$, and 2.51 in group B $(\mathrm{n}=4)$, for a group difference of $0.65 \pm 0.30, P=0.031$. Figure 1 shows confocal microscopic images at baseline (1A), CAC only (1B), and CAC-CAE (1C) at approximately $250 \times$ magnification. More inflammatory cells are evident in the subjects from group A who underwent the CAE exposure prior to the CAC.

\section{Discussion}

The results of this study demonstrate that in subjects with a history of both dry eye and ocular allergy, a break in the ocular surface has a direct effect on clinical reactions to allergens. When the corneal epithelium was compromised by a CAE challenge, subjects reacted not only with more severe itching, conjunctival and episcleral redness, and chemosis, but also with a more rapid response, showing peak redness and swelling at 7 minutes following CAC instead of the usual peak at 20 minutes. This rapid dilation might reflect a more vulnerable conjunctival blood vessel, a pathological state brought on by the coexistence of allergy and dry eye, and the many inter-related and cumulative events at the origin of both diseases.

Epithelial changes in the conjunctiva and its vasculature are thought to be intrinsic to allergic disease. In clinical biopsies from patients with out-of-season allergic conjunctivitis, altered structural and tight junction proteins were found in the quiescent conjunctival epithelium. ${ }^{17}$ Supporting this premise, a previous study we conducted in an animal model demonstrated that during the late phase of ocular allergy, expression of the proteins, ZO1 and E-cadherin, which maintain tight junctions between vascular epithelial cells, thereby preventing leakage, chemotaxis, and eventual cellular infiltration, were decreased significantly. ${ }^{18}$ This slackening of vessels might be accelerated by a dry eye challenge in susceptible subjects. Allergen challenge in humans has recently been shown to increase hyperemic neuromediators, such as substance $\mathrm{P}$ and VIP, ${ }^{19}$ and immunoreactive nerve terminals to VIP have been shown to be increased in Sjogren's syndrome. ${ }^{20}$

In the cytokine-rich environment of the ocular surface in dry eye, mast cells in the conjunctival epithelium appear to be hyperactivated. The corneal and conjunctival inflammation observed in this study after CAE might also have primed limbal mast cells, known to be abundant in the eye. ${ }^{21}$ A compromised ocular surface might allow more antigen to make contact with and bind to mast cells, resulting in a heightened allergic response, ie, a lower threshold response, in comparison with what occurred in these same subjects with a healthy ocular surface the week before. In eyes that had undergone the stress of the $\mathrm{CAE}$ challenge, which was shown before the $\mathrm{CAC}$ to have increased discomfort and fluorescein staining compared with subjects in a normal environment, inflammatory pathways were already activated by neurogenic input from the compromised corneal surface, resulting in a more rapid and cumulative conjunctival inflammatory response to allergen-induced mast cell degranulation. Supporting this ripple-down effect from the cornea to the conjunctiva, environmental desiccation stress in mice was shown to induce $\mathrm{T}$ cell-mediated inflammation of the cornea, conjunctiva, and lacrimal gland, suggesting that desiccation stress exposes shared epitopes in the these tissues. ${ }^{22}$ While preliminary in nature, the evidence provided by confocal microscopy does appear to corroborate a background of heightened inflammation in the CAE-challenged allergic subjects. These trends will be further delineated in future studies with a greater number of subjects.

The lack of an effect of CAE on TFBUT was not unexpected, because this parameter often appears to be independent of positive fluorescein staining, ie, the endpoint used in this study as clinical evidence of breaks in the ocular surface. Different subsets of dry eye exist, ${ }^{23,24}$ and it is possible that subjects with positive fluorescein staining as opposed to altered TFBUT were preselected since staining was an entrance inclusion criterion. It is also possible that under the desiccating conditions of CAE, reflex tearing might have occurred, albeit not therapeutically sufficient to diminish the observed corneal staining or the subsequent effect on the allergic response.

While the occasional patient may pose a diagnostic dilemma, dry eye and allergy are readily separated by signs and symptoms. The allergic eye has a moist, mildly pinkish-red, microchemotic glaze, and pathognomonic itch, while the dry eye has characteristic staining and redness predominantly in the horizontal vessels of the interpalpebral 
fissure. The patient with dry eye will complain of dryness, burning, and grittiness particularly in relation to visual tasking, and with a notable diurnal component, ${ }^{25}$ while symptoms of the allergic patient are independent of visual tasking.

In conclusion, in patients with both ocular allergy and dry eye, exposure to an adverse ocular environment prior to allergen challenge exacerbated the clinical response of itching, redness, and chemosis. This finding might have relevance to both the treatment and diagnosis of patients with these two diseases. It is not known if the exacerbation was due to a breakdown in the tear film barrier leading to higher allergen load of mast cells. Future studies are planned to identify the comorbidity of allergic conjunctivitis and dry eye in our study populations, to delineate further the conjunctival vessel changes after CAC through confocal microscopy, to identify if an adverse environment challenge would exacerbate ocular allergic signs and symptoms in normal subjects without dry eye, and to modulate endpoints of this dual challenge model with potential treatments for ocular allergy and/or dry eye.

\section{Disclosure}

The authors report no conflicts of interest in this work.

\section{References}

1. Singh KS, Axelrod S, Bielory L. The epidemiology of ocular and nasal allergy in the United States, 1988-1994. J Allergy Clin Immunol. 2010;126:778-783.

2. Lemp, MA. Advances in understanding and managing dry eye disease. Am J Ophthalmol. 2008;146:350-335.

3. American Academy of Ophthalmology. American Academy of Ophthalmology Corneal/External Disease Panel. Preferred Practice Pattern Guidelines. Dry Eye Syndrome - Limited Revision. San Francisco, CA: American Academy of Ophthalmology; 2011.

4. Schaumberg, DA, Sullivan DA, Buring JE, Dana MR. Prevalence of dry eye syndrome among US women. Am J Ophthalmol. 2003;136:318-326.

5. Berdy, GJ, Hedqvist B. Ocular allergic disorders and dry eye disease: associations, diagnostic dilemmas, and management. Acta Ophthalmol Scand. 2000;230 Suppl:32-37.

6. Hom MM, Nguyen AL, Bielory L. Allergic conjunctivitis and dry eye. Ann Allergy Asthma Immunol. 2012;108:163-166.

7. Stern ME, Pflugfelder SC. Inflammation in dry eye. Ocul Surf. 2004;2: 124-130.

Clinical Ophthalmology

\section{Publish your work in this journal}

Clinical Ophthalmology is an international, peer-reviewed journal covering all subspecialties within ophthalmology. Key topics include: Optometry; Visual science; Pharmacology and drug therapy in eye diseases; Basic Sciences; Primary and Secondary eye care; Patient Safety and Quality of Care Improvements. This journal is indexed on
8. Choi SH. Late-phase reaction in ocular allergy. Curr Opin Allergy Clin Immunol. 2008;8:438-444.

9. Trocme SD, Raizman MB, Bartley GB. Medical therapy for ocular allergy. Mayo Clin Proc. 1992;67:557-565.

10. Bonini S, Bonini S. IgE and non-IgE mechanisms in ocular allergy. Ann Allergy. 1993;71:296-299.

11. Leonardi A, Bogacka E, Fauquert JL, et al. Ocular allergy: recognizing and diagnosing hypersensitivity disorders of the ocular surface. Allergy. 2012;67:1327-1337.

12. Abelson MB, Chambers WA, Smith LM. Conjunctival allergen challenge. A clinical approach to studying allergic conjunctivitis. Arch Ophthalmol. 1990;108:84-88.

13. Abelson MB, Loeffler O. Conjunctival allergen challenge: models in the investigation of ocular allergy. Curr Allergy Asthma Rep. 2003;3: $363-368$.

14. Ousler GW, Gomes PJ, Welch D, Abelson MB. Methodologies for the study of ocular surface disease. Ocul Surf. 2005;3:143-154.

15. Patane MA, Cohen A, From S, Torkildsen G, Welch D, Ousler GW 3rd. Ocular iontophoresis of EGP-437 (dexamethasone phosphate) in dry eye patients: results of a randomized clinical trial. Clin Ophthalmol. 2011;5:633-643.

16. Semba CP, Torkildsen GL, Lonsdale JD, et al. A Phase 2 randomized, double-masked, placebo-controlled study of a novel integrin antagonist (SAR 1118) for the treatment of dry eye. Am J Ophthalmol. 2012;153: $1050-1060$

17. Hughes JL, Lackie PM, Wilson SJ, Church MK, McGill JI. Reduced structural proteins in the conjunctival epithelium in allergic eye disease. Allergy. 2006;61:1268-1274.

18. Ono SJ, Lane K. Comparison of effects of alcaftadine and olopatadine on conjunctival epithelium and eosinophil recruitment in a murine model of allergic conjunctivitis. Drug Des Devel Ther. 2011;5:77-84.

19. Sacchetti M, Micera A, Lambiase A, et al. Tear levels of neuropeptides increase after specific allergen challenge in allergic conjunctivitis. Mol Vis. 2011;17:47-52.

20. Batbayer B, Nagy G, Kovesi G, et al. Morphological basis of sensory neuropathy and neuromodulation in minor salivary glands of patients with Sjogren's syndrome. Arch Oral Biol. 2004;49:529-538.

21. McMenamin PG, Morrison SM, McMenamin C. Immunomorphologic studies of mast cell heterogeneity, location, and distribution in the rat conjunctiva. J Allergy Clin Immunol. 1996;97:1375-1386.

22. Niederkorn JY, Stern ME, Pflugfelder SC, et al. Desiccating stress induces T cell-mediated Sjögren's syndrome-like lacrimal keratoconjunctivitis. J Immunol. 2006;176:3950-3957.

23. Lam H, Bleiden L, de Paiva CS, et al. Tear cytokine profiles in dysfunctional tear syndrome. Am J Ophthalmol. 2009;147:198-205.

24. Toda I, Shimazaki J, Tsubota K. Dry eye with only decreased tear break-up time is sometimes associated with allergic conjunctivitis. Ophthalmology. 1995;102:302-309.

25. Walker PM, Lane KJ, Ousler GW 3rd, Abelson MB. Diurnal variation of visual function and the signs and symptoms of dry eye. Cornea. 2010;29:607-612.

\section{Dovepress}

PubMed Central and CAS, and is the official journal of The Society of Clinical Ophthalmology (SCO). The manuscript management system is completely online and includes a very quick and fair peer-review system, which is all easy to use. Visit http://www.dovepress.com/ testimonials.php to read real quotes from published authors. 